\title{
Attention bias modification training for adolescents with chronic pain: A randomized placebo-
}

controlled trial

Lauren C Heathcote ${ }^{1}$, Konrad Jacobs ${ }^{2}$, Dimitri M L Van Ryckeghem ${ }^{3,4}$, Emma Fisher ${ }^{5}$, Christopher Eccleston $^{3,6}$, Elaine Fox ${ }^{7}, \&$ Jennifer Y F Lau ${ }^{8 *}$

1 Department of Anesthesiology, Perioperative, and Pain Medicine, Stanford University, CA, USA

2 Oxford Centre for Children and Young People in Pain (OxCYPP), Nuffield Orthopaedic Centre, Oxford, United Kingdom

3 Department of Experimental-Clinical and Health Psychology, Ghent University, Ghent, Belgium

4 Institute for Health and Behaviour, INSIDE, University of Luxembourg, Luxembourg

5 Centre for Child Health, Behavior, and Development, Seattle Children's Research Institute, Seattle, WA, United States

6 Centre for Pain Research, University of Bath, Bath, United Kingdom

7 Department of Experimental Psychology, University of Oxford, Oxford, United Kingdom

8 Department of Psychology, King's College London, London, United Kingdom

*Corresponding author: Jennifer Lau, Department of Psychology, Institute of Psychiatry, Psychology, and Neuroscience, King’s College London, 16 De Crespigny Park, London, SE58AF, United Kingdom. Tel: +44 (0) 207848 0002. Electronic mail may be sent to jennifer.lau@kcl.ac.uk.

This study was funded by a Research Training Fellowship awarded to Lauren Heathcote by Action Medical Research for Children (Ref: GN2122). Elaine Fox is supported by an ERC Advanced Investigator Award (Ref: 324176). There are no conflicts of interest arising from this work.

Category: Clinical trial

Number of text pages: 33

Number of tables: 3

Number of figures: 2

Keywords: attention bias modification, cognitive bias, pediatric chronic pain, randomized controlled trial 


\section{INTRODUCTION}

Attending toward and focusing on pain are central components in many psychological models of chronic pain $[25,26,48,58,62]$. In particular, an 'attentional bias' toward pain, the tendency to select pain-related information over non-pain information, is argued to increase the salience of pain, enhance fear and catastrophizing, motivate avoidance of painful activities, and increase disability. Given this crucial role, studies have begun to investigate whether pain-related attention biases can be modified and whether this modification impacts pain and pain-related outcomes. One tool for this purpose is Attention Bias Modification (ABM), a computerized training protocol that is proposed to train participants' attention away from pain-related stimuli, possibly by altering topdown attention control $[5,35]$.

ABM has been shown to increase cold pressor pain thresholds in healthy adults $[3,43,54]$, and to significantly reduce pain intensity and frequency in adults with acute back pain [53]. In chronic pain samples, one uncontrolled study [52] and one placebo-controlled study [9] provided evidence that $\mathrm{ABM}$ decreases pain symptoms, although a third placebo-controlled study did not replicate these findings [53]. Studies with chronic pain samples have also provided evidence that ABM significantly reduces pain-related outcomes such as anxiety sensitivity [43,53], functional disability [53], and anxiety and depression symptoms [52]. Taken together, there is preliminary evidence that ABM may be efficacious for improving pain and pain-related outcomes, although findings across studies are mixed and additional, randomized-controlled trials are needed [59].

No studies have yet employed ABM for adolescents with chronic pain, despite previous findings that selective attention biases characterize paediatric patients with chronic pain $[2,4]$ and that therapeutic techniques targeting attention, particularly attention control can moderate adolescents' cognitive-affective response to pain as well as pain outcomes [33,34]. Strategies such as $\mathrm{ABM}$ that can encourage improvements in attention control ability - particularly at a developmental juncture when there are age-typical changes in mastery over attention $[15,44]$ and 
associated maturation of brain regions engaged in goal-directed attention control $[44,46,57]$ - may be especially beneficial. In addition, as chronic pain often first emerges in adolescence [47], investigating the plasticity and impact of pain-related attention during adolescence may allow consideration of its role in the early emergence and maintenance of chronic pain.

Here we report a three-arm randomized, placebo-controlled, parallel-group trial of multisession $\mathrm{ABM}$ in adolescents with chronic non-cancer pain. We predicted that $\mathrm{ABM}$, relative to placebo training and no training (waitlist), would significantly reduce attention bias to pain-related stimuli while also increasing attention control. We also expected that ABM would lead to relative improvements in pain symptoms and pain-related outcomes, namely pain catastrophizing, anxiety and depression symptoms, and functional disability. We examined effects of ABM on pain and pain-related outcomes immediately after training and three months later, to examine whether training effects maintained or emerged over time.

\section{METHODS}

This study was registered on the NIHR Clinical Research Network Portfolio in August 2014 (UK Clinical Trials Gateway: CPMS 17251).

\section{Participants}

Adolescents aged 10-18 years with chronic non-cancer pain were recruited from the Oxford Centre for Children and Young People in Pain (OxCYPP), part of the Nuffield Orthopaedic Centre at the Oxford University Hospitals. OxCYPP is part of an orthopaedic musculoskeletal referral system receiving referrals from gastroenterology, neurology, orthopaedics, spinal units, rheumatology, and General Practitioners. Patients were eligible for the current study if they reported recurrent or persistent pain for more than three months. Participants were excluded if they were currently experiencing severe distress based on expert clinician judgment by a consultant 
clinical psychologist, or did not understand English. Patients were only eligible to take part in the study if they were not scheduled to receive multidisciplinary inpatient treatment, or regular psychological treatment, during the intervention (training) phase. However, patients were permitted to continue attending infrequent (once per week or less) outpatient sessions, including with the occupational therapist or physiotherapist, primarily for assessments. Some patients were also seeking additional complementary and alternative treatments outside of the hospital setting, including acupuncture and nutritional support. These treatments were also permitted if they were being received infrequently (once per week or less). We recruited participants between October 2014 and April 2016, and the final assessment session took place in August 2016. Participants were recruited primarily for the current study investigating $\mathrm{ABM}$ training, but we also collected measures of interpretation bias at baseline (data reported elsewhere; [32]). Patients and a parent/guardian were first approached about the study either following their first assessment session at the clinic, or following their second visit to the clinic during which they attended a pain education class with a small group of other patients and families. During these sessions, a paediatric rheumatologist or the consultant clinical psychologist approached patients and their families and asked if they would like to learn more about taking part in a research study. A member of the research team subsequently provided the families who were happy to be approached with an information sheet, answered questions about the study, and scheduled appointments. Seventy-three patients were interested in the study, and 67 agreed to take part. Reasons for not participating in the study were not recorded for patients who were approached but declined to take part. A CONSORT diagram illustrating the flow of participants through each stage of the trial is shown in Figure 1. The first patient was recruited as a pilot participant and only completed a small number of measures to examine feasibility of testing in the hospital setting, and so the data from this patient were excluded from analyses. Thus, 66 patients were enrolled in the full study (55 female; $M=$ 
13.97 years; $S D=2.13$ ). This study was approved by the UK National Research Ethics Service (REC reference 14/SC/0246).

\section{Procedures}

Recruitment and assessment sessions took place at the Nuffield Orthopaedic Centre. At the baseline assessment session, parents/guardians first provided informed consent for their children. In addition, participants aged 16 or over provided informed consent for themselves. Participants younger than 16 years provided informed assent. Randomisation took place following consent.

\section{Randomisation and Allocation Concealment}

Eligible participants were randomly assigned to one of the three treatment arms (ABM, placebo, or waitlist), with an equal allocation ratio, based upon a computerized random number generator (www.random.org/). The principal investigator (LCH) performed randomisation following successful recruitment and consent, thus allocation to treatment arm was fully concealed to participants and to the research team prior to assignment.

\section{Blinding}

Participants assigned to ABM or placebo training were blinded to their treatment condition throughout the trial. Given that participants assigned to the waitlist condition were told that they would not receive the training tasks until after the final assessment session, it was not possible to blind participants assigned to the waitlist condition. Study physicians involved in the participants' otherwise normal care were blind to allocation. Only the principal investigator (LCH), who conducted all assessment sessions, was aware of the participants' treatment conditions out of necessity for sending participants the correct training protocol. 


\section{Training Protocols}

Participants in the $\mathrm{ABM}$ and placebo training groups were asked to complete eight training sessions, twice per week over four weeks. Participants were encouraged to do the training during normal waking hours, and to allow at least one day between the sessions. SMS reminders were sent to participants, parents, or both depending on the family's preference. Participants in the waitlist group did not complete any training between the baseline and post-training assessment, but were given the opportunity to complete the training after the final (follow-up) assessment session.

\section{Assessment Sessions}

At the baseline assessment session, we collected demographic information and clinical history. At all assessment sessions (baseline, post-training, and follow-up) participants completed the measure of pain symptoms (primary outcome), followed by the measures of attention bias and

attention control, physical tests (as an index of functional disability), and finally self-reported functional disability, pain catastrophizing, and anxiety and depression symptoms. The post-training assessment was completed within seven days of completing the last training session, and the follow-up assessment was completed three months after the post-training assessment. In addition to the measures described above, participants completed two additional questions at the post-training assessment to provide subjective feedback on the training. Participants were compensated with an Amazon voucher after each assessment session (vouchers worth $£ 100$ in total), and travel expenses were reimbursed to parents. All adverse events, regardless of whether they were study-related, were monitored throughout and reviewed by the principal investigator ( $\mathrm{LCH})$ at each study assessment.

\section{ABM and Placebo Training}

$\mathrm{ABM}$ and placebo training were delivered using modified versions of the dot-probe task [39] (see Figure 2). During ABM and placebo training, stimuli were presented against a black 
background. Each trial began with a 500ms presentation of a white fixation cross in the middle of the screen. Participants were instructed to fixate their gaze on this location. Then, one stimulus pair comprising a pain-related stimulus and a neutral stimulus appeared and remained visible for 500ms (for a similar protocol see [53]). One stimulus was presented above and one below the fixation cross, with the distance from the stimuli to the centre of the screen as one-fourth the height of the screen. Immediately after the offset of these two stimuli, a letter 'p' or 'q' would appear in place of one of the stimuli (the probe). During ABM training, the probe always appeared in the previous location of the neutral stimulus, and stimulus pairs were randomly presented in each of the two possible combinations (probe up/pain stimulus down; probe down/pain stimulus up). Hence, the aim of the ABM training was developed to train participants to attend away from pain-related stimuli. During placebo training, the probe appeared equally often in the location of the painrelated stimulus and the neutral stimulus, and thus stimulus pairs were randomly presented in each of the four possible combinations (probe up/pain stimulus up; probe up/pain stimulus down; probe down/pain stimulus down; probe down/ pain stimulus up). In both training conditions, participants had to indicate whether the probe was a 'p' or a 'q' by pressing the corresponding button on the (QWERTY) keyboard as quickly as possible. The 'q' key was pressed with the left index finger and the 'p' key was pressed with the right index finger. A new trial started after each response, or automatically when $2500 \mathrm{~ms}$ elapsed without response. When a participant responded erroneously, the term 'error' briefly appeared on the screen $(200 \mathrm{~ms})$. In order to ensure that participants maintained gaze at the middle of the screen, a number of digit trials were presented (see e.g., $[34,51])$. In these trials, the fixation cross was followed by a random digit between 1 and 9 for a duration of $150 \mathrm{~ms}$. Participants were instructed to type the number on the keyboard. The inter-trial interval was $1000 \mathrm{~ms}$. In the context of the current study, congruent trials were those where the probe was presented at the same location as the pain stimulus. Incongruent trials were those where the probe was presented at the same location as the neutral stimulus. Each training session 
consisted of 210 training trials (96 congruent trials, 96 incongruent trials, 18 digit trials), taking 12 minutes for participants to complete. Stimuli were presented in a randomized order across trials and participants, and trials were intermixed and randomly presented in two blocks, with a break between block one and block two.

The dot-probe tasks were programmed and presented using the INQUISIT Millisecond software package (INQUISIT 4.0). INQUISIT measures response times (RTs) with millisecond accuracy (Inquisit 4.0; Millisecond Software, Seattle, WA, USA). Participants completed all ABM and placebo training at home, using the INQUISIT Web interface. This interface allows participants to access each training task using a unique webpage address. The INQUISIT programme is downloaded to the participant's local computer using a plugin. Each time the participant finished the task, a data-file with the participant's accuracy and response time scores is securely saved to the experimenter's INQUISIT account, allowing the experimenter to verify that the participant had completed each training task, and to monitor the participant's performance.

\section{Measures}

All primary and secondary outcome measures, and measures of attention bias and attention control, were completed at baseline, post-training assessment, and follow-up assessment. Details of training tasks and assessment measures are given below.

\section{Demographics}

At baseline, participants reported on demographic information including gender, age, ethnicity, school status, and number of siblings. Participants also reported on the onset of their pain (sudden or gradual), time since pain onset (months), and body locations where they experience pain. 


\section{Primary Outcome: Pain Symptoms}

To assess pain symptoms, participants completed two 11-point visual analogue scales indicating their average pain intensity in the last week, and their pain intensity at the body part they experienced most problems within the last week (hereafter referred to as 'most problematic pain') $(0=$ no pain; $10=$ worst pain possible), as well as one 4-point visual analogue scale indicating the average duration of their pain each day in the last week $(1=$ less than one hour; $4=$ all day $)$. All items were derived from the Brief Pain Inventory [14].

\section{Secondary Outcomes}

\section{Physical Disability}

Participants' physical disability was assessed with one self-report measure and two physical tasks. The self-report measure (FDI) was the primary measure of physical functioning.

First, participants completed the Functional Disability Inventory (FDI) that assesses perceived difficulty in performing common activities in the domains of school, home, recreation, and social interactions. Participants rate the difficulty they had in carrying out each activity in the preceding two weeks $(0=$ no trouble; $4=$ impossible $)$. The FDI consists of 15 items and yields a total score that can range from 0 to 60 , with higher scores indicating greater disability. The FDI has yielded good reliability and validity for children and adolescents [63] and for youth with chronic pain [37]. Cronbach's alpha for our sample was .90 at baseline.

Second, two objective assessments of physical function were conducted. Participants completed the Sit-to-Stand task, which comprised participants attempting to move from a seated to a standing position as many times as possible within 30 seconds. Participants also completed the Cardio Wall task (http://embeddedfitness.nl/en/exergames/cardio-wall-2/), an interactive task in which participants are required to tap (using their hands) lights on a wall for 30 seconds. 
Participants must reach high and bend down low to tap the lights, and the maximum height can be adjusted according to the height of the participant.

\section{Pain Catastrophizing}

We assessed participant's catastrophic thoughts about pain using the Pain Catastrophizing Scale for Children (PCS-C) [18]. Pain catastrophizing is comprised of rumination, magnification, and helplessness. Participants rated 13 items on a $0-4$ scale to create a total score from 0 to 52 . Higher scores indicate more pain catastrophizing. The PCS-C has good reliability and validity for children with chronic pain above 9 years [18]. Cronbach's alpha for our sample was .88 for the total score at baseline.

\section{Generalized Anxiety and Depression}

Generalized anxious and depression symptomatology was assessed using the Revised Child Anxiety and Depression Scale (RCADS) [11]. The RCADS consists of 47 items which are scored on a 4-point Likert scale from never to always. Higher scores indicate increased symptoms of anxiety and depression. The RCADS comprises six subscales but here we only calculated scores for Generalized Anxiety Disorder (GAD) and Major Depression Disorder (MDD) as we were interested in whether $\mathrm{ABM}$ training would improve general anxiety and depression symptomatology, rather than symptoms related to other anxiety disorders such as separation anxiety or social anxiety. The RCADS has yielded good reliability and validity for children and adolescents $[10,28,42]$. Cronbach's alpha in this study was .86 for the GAD subscale and .86 for the MDD subscale at baseline.

\section{Attention Bias Measurement (dot-probe) Task}


The dot-probe task used to measure attention bias was similar to the placebo training tasks (described above), in which participants were presented with stimulus pairs comprising one painrelated and one neutral stimulus, and were required to identify a probe (a letter ' $p$ ' or ' $q$ '). Only child picture stimuli (not adult faces or pain words) were used in the attention bias measurement task. Like in the placebo training, the pain-related stimuli and probe were presented equally often at the top or bottom position of the screen in four possible combinations: pain stimulus top/probe top; pain stimulus top/probe bottom; pain stimulus bottom/probe bottom; pain stimulus bottom/probe top. Thus, the probe was equally likely to replace either a pain-related stimulus or neutral stimulus. Stimuli were presented in a randomized order across trials and participants. Trials were intermixed and randomly presented in two blocks, with a break between block one and block two. However, unlike the training tasks, each measurement task also began with 20 practice trials comprising 16 neutral stimulus-pairs, none of which appeared in the experimental trials, as well as four digit trials. Thus, in total, each test phase (baseline, post-training, and follow-up) comprised 274 trials (96 congruent trials, 96 incongruent trials, 64 neutral-neutral trials, 18 digit trials), taking 12 minutes for participants to complete. Neutral-neutral trials were included as filler trials to reduce possible habituation to pain-related stimuli that might occur if all trials contained pain-related information [34]. Participants completed all attention bias measurement tasks at the hospital, using the INQUISIT Lab programme on an experimenter-provided computer.

\section{Attention Control Measures}

Participants' levels of attention control were assessed with one self-report measure and one experimental measure.

The Attention Control Scale (ACS) [23] is a self-report questionnaire that comprises 20 items, and yields a total score that can range from 20 to 80 , with higher scores indicating good attention control. The ACS has two subscale scores for attention focusing and attention shifting. 
The ACS has shown good reliability and predictive utility, predicting resistance to interference in Stroop-like spatial conflict tasks as well as attentional disengagement from threat stimuli among highly anxious people [23]. Furthermore, Matthews and colleagues [41] showed greater activation in brain areas related to top-down regulation of emotion (i.e., rostral anterior cingulate cortex, rACC) in those reporting greater attention control. Attention control has also been measured with good reliability and validity in children [45]. Cronbach's alpha in our sample was .81 for the total score at baseline.

The Fish Flanker Task [12,49] is an experimental measure of attention control developed by Christ and colleagues [12]. For this task, participants completed a series of computerized trials in which they saw a horizontal row of five fish. On each trial, participants were asked to respond as quickly as possible as to whether the centre fish was facing to the left or right (using the corresponding arrow keys on the computer keyboard). Response times were measured. Two trial types were administered: congruent and incongruent. On congruent trials, all five fish in the stimulus array pointed in the same direction. On incongruent trials, the four distracting fish pointed in the opposite direction of the central target fish. For each trial, stimuli were presented until a response was made or until more than $3000 \mathrm{~ms}$ elapsed. After an inter-trial interval of $1500 \mathrm{~ms}$, a new trial was presented. If participants failed to respond within $3000 \mathrm{~ms}$, a tone and a message stating "Too slow" were presented. If participants pressed the incorrect arrow button, a tone and a message stating "Wrong response" were presented. Participants completed 120 experimental trials (60 congruent and 60 incongruent trials) that were randomly intermixed. Presentation was balanced such that all possible stimulus-flanker pairings were equally likely to occur. At intervals of 40 trials, children were offered a timed 30 second break. Response times on congruent and incongruent trials comprised outcome variables for this task.

\section{Subjective Feedback on Training}


At the post-training assessment, participants completed two six-point visual analogue scales $(0=$ Not at all; $5=\mathrm{A}$ lot $)$ to gauge their enjoyment in completing the training ('How much did you enjoy doing the training?') and the degree to which participants thought the training helped them ('How much do you think the training helped you?').

\section{Materials}

\section{Dot-Probe (Attention Bias) Training and Measurement Stimuli}

Three sets of picture stimuli and one set of word stimuli were used in the ABM training tasks. Only picture stimuli were used in the dot-probe assessment tasks, whereas a mixture of picture and word stimuli were used for training.

Stimulus Set One was used at the baseline assessment session and for two of the training sessions. Stimulus Set Two was used at the post-training and follow-up assessment sessions, but not during the training. Thus, attention biases were examined at the post-training and follow-up assessments for non-trained stimuli. For the test phase, both stimulus sets comprised 16 facial image stimuli, taken from 8 children (four male and four female). For each stimulus set, 8 picture pairs were created. Each pair comprised two pictures of the same child. For each set, in two of the pairs the child presented a neutral face in both pictures. These picture pairs were used as filler trials (filler trials were not included during the training phase; see [34]). In the remaining 12 picture pairs (6 pairs per set), there was one picture with the child presenting a neutral face and one picture with the child presenting a pain face. All pain pictures were drawn from video clips of children taking part in a cold pressor pain experiment at Ghent University. Stimuli were $160 \times 264$ pixels in size, presented on an Apple Macbook Pro with a 13-inch screen. As these child stimuli were not previously validated, we asked participants at the post-training assessment session to rate each child face for pain intensity using a 0-10 Numeric Rating Scale (NRS) (i.e., 'How much pain has 
the child displayed on the picture; anchors; 'no pain at all'; 'a lot of pain') (see Supplementary Materials A for results confirming that stimuli were attributed correctly).

Stimulus Set Three was used for two of the training sessions. It comprised 12 facial image stimuli, presenting six adult faces (three male and three female). Stimuli were 160 x 264 pixels in size. All pictures were drawn from one-second video clips of simulated facial expressions of pain. These pictures were taken from a larger collection of stimuli, previously created and validated in the laboratory by Simon and colleagues [56]. For these stimuli actors were videotaped while producing neutral facial displays and simulated facial expressions of different pain intensities. Using these 12 pictures, six study slides were generated. Each slide consisted of two pictures of the same adult, presenting a neutral face or a moderate pain face. These stimuli have been used in prior research examining attention bias to pain (see [60]). The validity of this stimulus set used is supported by previous findings of significantly different observer pain ratings between neutral and moderate pain facial expressions (see [61]).

Stimulus Set Four was used for four of the training sessions. The total stimulus set comprised 54 pain/neutral word pairs which were matched for length and frequency (see Supplementary Materials B for full list of words). However, only 12 word pairs were selected for each participant. These pairs were selected in order to best match the quality of pain experienced by each individual participant. Therefore, word stimuli were idiosyncratically matched for each participant. To select the 12 word pairs for each participant, participants were provided with the list of 54 pain-related words at baseline, and were asked to indicate how well each word described his/her pain (VAS: $0=$ Not at all; 5=Perfectly). Based on these ratings, we first selected words with the highest ratings (i.e., 5 out of 5). Next, we selected words with successively lower ratings until a total of 12 word-pairs were selected. If more than 12 words were possible to select (i.e., with the highest ratings), we selected the words with the shortest length. 


\section{Statistical Analysis Plan}

Incomplete Outcome Data

Per-Protocol (PP) and Intention-to-Treat (ITT) analyses were conducted. Following guidance from the Committee for Proprietary Medicinal Products (CPMP), the ITT analysis is considered primary and the PP analysis is considered supportive [31] (PP analysis is presented in Supplementary Materials C). Regarding ITT analysis, we used the Baseline Observation Carried Forward (BOCF) method. Five participants (7.58\% of the full sample) dropped out of the study at either the post-training or follow-up assessments, and thus did not provide complete outcome data (see Figure 1 for details). For those participants in attendance at each session, any questionnaires in which participants did not complete at least $75 \%$ of items are removed for that participant. Mean imputation using the relevant subscale for the individual participant's score is used for questionnaire items where less than $25 \%$ of the scale is missing.

\section{Sample Size Determination}

Given that there are no similar published studies on ABM in youth with chronic pain, nor on $\mathrm{ABM}$ in adults with chronic pain that compare three groups (i.e., ABM, placebo, and waitlist), a minimum sample size of $N=20$ in each group was justified a priori on the basis that 1) this exceeds the sample size determined by Sharpe and colleagues [53] for their study of ABM in adults with chronic pain, for which sample size estimations revealed that $n=12$ participants per group was necessary to achieve $82 \%$ power with a significance level of 0.05 (based on findings of an effect size of 0.79 from [40]), 2) this exceeds the sample size determined by Eldar and colleagues [27] for their study of ABM in youth with anxiety disorders ( $n=10-15$ in each group), and 3) Simmons and colleagues [55] suggest a minimum of $N=20$ in each group to reduce the likelihood of falsepositive findings. 
Furthermore, earlier ABM studies of adult chronic pain reported large within-group comparisons across pain severity indices from pre- to post-intervention, from 0.88 (measure of total pain in Schoth et al, 2013) to 1.27 (visual analogue scale of current pain severity in Carleton et al, 2011). The more recent ABM trial reported by Sharpe and colleagues (2001), which did use a control group, reported a between-group effect size of 0.92 on average pain in their first study, and a within-group effect size of 0.49 on pain disability in their intervention group in the second study. To the extent that these effect sizes are generalizable to our sample and design, $n=20$ in our intervention and control-training group should provide $80 \%$ power to detect these effects at $\mathrm{p}<0.05$ (two-tailed).

\section{Analyses}

All analyses were conducted using SPSS 23.0 software. We first performed a series of one-way ANOVAs and chi-square tests to determine whether there were any differences between the three randomized groups (ABM, placebo, waitlist) on demographic variables, outcome variables, and task measures at baseline. Next, we performed $t$-tests to determine if participants demonstrated a significant attention bias towards pain at baseline, followed by a 2 (Congruency: congruent, incongruent) x 3 (Time: baseline, post-treatment, follow-up) x 3 (Group: ABM, placebo, waitlist) ANOVA on attention bias and attention control indices to establish whether there was a training effect. The main analyses comprised a series of 3 (Time: baseline, post-treatment, follow-up) x 3 (group: ABM, placebo, waitlist) ANOVAs for outcome variables. If analyses yielded any significant interaction effects which, when decomposed, revealed a significant effect of ABM training compared to placebo or waitlist, we planned to perform Pearson correlation analyses to assess whether change in attention bias or attention control were associated with change in the outcome variable. If so, formal mediation analyses were planned. 
If the sphericity assumption was violated across multiple ANOVA tests (i.e., if Mauchly's test of sphericity was $p<0.05$ ), Greenhouse-Geisser corrections (with adjusted degrees of freedom) would be performed and reported for tests. Of note, due to technical difficulties with INQUISIT, dot-probe (attention bias) data were unavailable for one participant (in the waitlist group) at baseline. Data from this participant were retained in all other analyses.

\section{Dot-Probe Task Data Preparation}

Digit trials were first discarded. Consistent with previous research [7,34,51,61], trials with errors, and responses shorter than $200 \mathrm{~ms}$ or longer than $2000 \mathrm{~ms}$ were also discarded. Within the present sample, the number of errors made by participants ranged from 2-30 $(M=11.06)$ for the baseline assessment, from 1-73 $(M=11.63)$ for the post-training assessment, and from $1-36(M=$ 9.20) for the follow-up assessment. After discarding these error trials, $0.2 \%$ of response times (RTs) in the remaining dataset at baseline, $0.4 \%$ of RTs in the remaining dataset at post-training, and $0.2 \%$ of RTs in the remaining dataset at follow-up fell outside the range of $200 \mathrm{~ms}$ to $2000 \mathrm{~ms}$. Probe detection latencies that were three SDs above or below the individual mean RT of correct responses for each trial type were also considered outliers and thus excluded from analyses $[7,34,38,61]$. This was the case for $1.4 \%$ of the RTs in the remaining dataset at baseline, $1.6 \%$ of the RTs in the remaining dataset at post-training, and $1.7 \%$ of the RTs in the remaining dataset at follow-up. Filler (neutral/neutral trials) were also discarded before means and SDs were calculated.

\section{Fish Flanker Task Data Preparation}

Consistent with previous research [12], trials with errors, and responses shorter than 200ms or longer than $3000 \mathrm{~ms}$, were discarded. Within the present sample, $2 \%$ of trials were removed from the baseline assessment, $2.3 \%$ of trials were removed from the post-training assessment, and $2.3 \%$ of trials were removed from the follow-up assessment. As with the dot-probe data preparation, RTs 
that were three SDs above or below the individual mean RT of correct responses for each trial type were also considered outliers and thus excluded from analyses. This was the case for $1.7 \%$ of the RTs on congruent trials and $1.7 \%$ of the RTs on incongruent trials in the remaining dataset at baseline, $1.8 \%$ of the RTs on congruent trials and $1.8 \%$ of the RTs on incongruent trials in the remaining dataset at the post-training assessment, and $1.9 \%$ of the RTs on congruent trials and $1.6 \%$ of RTs on incongruent trials in the remaining dataset at the follow-up assessment.

\section{RESULTS}

\section{Participant Characteristics}

Participant characteristics are presented in Table 1. Time since pain onset varied between 5 and 170 months $(M=45.7$ months, $M d n=35.5$ months $)$. Most participants reported multiple pains, with the most common pain problems including joint pain $(N=36,54.5 \%)$, pain in legs or feet $(N=$ $35,53 \%)$, back pain $(N=33,50 \%)$, and pain in hands or arms $(N=30,45.5 \%)$. Twelve participants $(18.2 \%)$ reported pain all over their body. When asked to indicate the body location where participants experienced the most pain, they most often indicated their back $(N=16,24.2 \%)$, legs or feet $(N=15,22.7 \%)$, or joints $(N=10,15.2 \%)$. Participants reported moderate levels of disability (as indexed by clinical reference points for the FDI; $M=22.79, S D=10.74$, [37]). Fortyfour participants $(66.7 \%)$ reported experiencing pain every day in the last three months, $20(30.3 \%)$ reported pain on most days, and two (3\%) reported pain on about one day per week. Thirty-one (47\%) participants reported that they had taken pain medication at some point in the weeks leading up to the study. Randomisation resulted in a balanced distribution across all conditions at baseline, with no significant group differences on demographic, outcome, and task assessment measures (see Tables $1 \& 2$ ). As only one participant dropped out of the study before completing the training, we were unable to examine whether there were any significant baseline differences between that participant and those participants who completed training. However, visual inspection of mean 
scores indicates that the participant who dropped out was similar across task and outcome measures.

\section{Treatment Adherence}

Out of the 45 participants who commenced either ABM or placebo training, 25 participants (55.6\%) completed all eight training sessions as scheduled. Of the remaining participants, all participants completed five or more training sessions with the exception of one participant who dropped out before completing any training sessions (participant excluded from PP analyses).

\section{Intention to Treat Analyses}

\section{Attention Bias Analyses}

Attention Bias at Baseline

There were no significant differences between RTs to congruent or incongruent trials at baseline $(t(64)=0.33, p=.74)$, and the calculated attention bias score was not significantly different from zero at baseline $(t(64)=-0.33, p=.74)$, indicating no attentional biases to painrelated stimuli across the whole sample (see Table 2 for means and SDs).

\section{Change in Attention Bias Following Training}

A 2 (Congruency: congruent, incongruent) x 3 (Time: baseline, post-treatment, follow-up) x 3 (Group: ABM, placebo, waitlist) ANOVA was conducted for dot-probe scores to assess whether a reliable training effect of ABM could be determined (see Table 2 for means and SDs; see Table 3 for ANOVA results). Analyses revealed a significant main effect for time, indicating that all participants, regardless of training, had faster RTs with proceeding time. Bonferroni-corrected analyses of simple main effects revealed that RTs were significantly faster at each assessment session (baseline to post-training: $p<.001$; post-training to follow-up: $p=.01$; baseline to follow- 
up: $p<.001)$. There were no other significant main effects, and no significant interactions effects. Thus, the predicted training effect was not observed.

\section{Primary Outcome Measures}

A series of 3 (Time: baseline, post-treatment, follow-up) x 3 (Group: ABM, placebo, waitlist) ANOVAs were conducted on pain symptoms (see Table 2 for means and SDs; see Table 3 for ANOVA results). There was a significant main effect of time for pain duration, indicating that all participants, regardless of training, experienced pain for less time each day with proceeding time. Bonferroni-corrected analyses of simple main effects revealed that participants experienced significantly less pain at follow-up compared to baseline $(p=.01)$ but not compared to posttraining (SPSS adjusted $p=1.0)$, nor at post-training compared to baseline $(p=.09)$. There were no significant main effects for average pain or most problematic pain, and contrary to our hypotheses, there were no significant interaction effects. Due to the absence of significant group differences on primary measures, effect sizes (with $95 \%$ confidence intervals) were thought to be important for planning future studies, specifically regarding sample size considerations. We therefore calculated effect sizes of differences between the $\mathrm{ABM}$ and placebo group at post-intervention for: pain at most problematic site, -0.127 (95\% CI: -0.712 to 0.458 ); average pain, -0.536 (95\% CI: -1.131 to 0.05); and pain duration, 0.221 (95\% CI: -0.365 to 0.807$)$.

\section{Secondary Outcome Measures}

A series of 3 (Time: baseline, post-training, follow-up) x 3 (Group: ABM, placebo, waitlist) ANOVAs were conducted on secondary outcome measures (see Table 2 for means and SDs; see Table 3 for ANOVA results). There were main effects of time for functional disability (baseline to post-training: $p<.001$; baseline to follow-up: $p<.001$; post-training to follow-up: $p=.04$ ), pain catastrophizing (baseline to post-training: $p<.001$; baseline to follow-up: $p<.001$; post-training 
to follow-up: $p=.12$ ), generalized anxiety (baseline to post-training: $p=.04$; baseline to followup: $p=.01$; post-training to follow-up: $p=.40$ ), depression symptoms (baseline to post-training: $p$ $=.37$; baseline to follow-up: $p=.04$; post-training to follow-up: $p=.21$ ), sit-to-stand task (baseline to post-training: $p=.03$; baseline to follow-up: $p=.002$; post-training to follow-up: $p=$ .12 ), and cardio wall task (baseline to post-training: $p<.001$; baseline to follow-up: $p<.001$; post-training to follow-up: $p=1.0$ ), indicating that participants reported improved scores over time on these measures, regardless of training group. There were no other significant main effects, and there were no significant interaction effects. As for primary outcomes, effect sizes (with $95 \%$ confidence intervals) of differences between the ABM and placebo group at post-intervention were calculated for: functional disability, 0.032 (95\% CI: -0.552 to 0.617$)$; pain catastrophizing, -0.147 (95\% CI: -0.733 to 0.438 ), generalized anxiety, -0.084 (95\% CI: -0.669 to 0.501 ), depression symptoms, 0.084 (95\% CI: -0.501 to 0.669 ), attention control, -0.230 (95\% CI: -0.817 to 0.356 ), sit-to-stand task, 0.366 (95\% CI: -0.224 to 0.955$)$, and cardio wall task, 0.213 (95\% CI: -0.373 to $0.800)$.

\section{Attention Control Analyses}

\section{Change in Attention Control after Training}

To examine whether ABM training changed self-reported attention control, we conducted a 3 (Time: baseline, post-training, follow-up) x 3 (Group: ABM, placebo, waitlist) ANOVA with the Attention Control Scale (ACS) score as the outcome variable (see Table 2 for means and SDs; see Table 3 for ANOVA results). There was a significant main effect of time, indicating that that all participants, regardless of training, reported higher attention control over time. Bonferronicorrected analyses of simple main effects revealed that participants reported significantly higher attention control at follow-up than at baseline $(p=.02)$. Self-reported attention control did not 
significantly differ between baseline and post-training $(p=.15)$ or between post-training and follow-up $(p=.49)$. There were no significant interaction effects.

To examine whether ABM training changed experimentally-measured attention control, we conducted a 2 (Congruency: congruent, incongruent) x 3 (Time: baseline, post-treatment, followup) x 3 (Group: ABM, placebo, waitlist) ANOVA for RTs on the flanker task (see Table 2 for means and SDs; see Table 3 for ANOVA results). There was a significant main effect of congruency, indicating that participants were slower on incongruent than congruent trials $(p<$ .001). There was also a significant main effect of time, indicating that participants, regardless of training, had faster RTs with proceeding time. Bonferroni-corrected analyses of simple main effects revealed that participants were significantly faster at follow-up compared to baseline $(p=.01)$ and at follow-up compared to post-training $(p=.01)$. Participant RTs did not significantly differ between baseline and post-training $(p=1.0)$. There were no other significant main effects, and no significant interactions effects.

\section{Adverse Events}

No adverse events reported by participants were study-related. No participants reported adverse events immediately following the baseline session. Eight participants reported adverse life events at the post-training assessment, and eight participants reported adverse life events at the follow-up assessment. These were flu $(N=1)$, illness or death of a non-immediate family member $(N=3)$, visits to hospital emergency departments $(N=1)$, accident-related injury $(N=1)$, parent in accident $(N=1)$, minor medical procedures $(N=1)$, difficulties at school $(N=3)$, and death of a pet $(N=1)$. Eight of these participants also reported pain or other physical symptoms as a specific adverse event; however, these were reported as unrelated to the study procedures. There were no significant differences in the number of adverse events reported between groups. 


\section{Subjective Feedback}

Participants who completed ABM or placebo training reported that they moderately enjoyed completing the training $(M=3.58$, Range $=2-5)$, and that they believed the training moderately helped them $(M=2.66$, Range $=0-5)$. When asked to predict whether they received $\mathrm{ABM}$ or placebo training, 29 participants $(65.9 \%)$ thought that they had completed placebo training, and there was no significant difference between groups in predicted training received (ABM group: 16 (72.7\% placebo); placebo group (13 (59.1\% placebo); $\left.X^{2}(1)=0.91, p=.34\right)$.

\section{DISUCSSION}

This study presents a randomized placebo-controlled trial of multi-session Attention Bias Modification (ABM) for adolescents with chronic pain. We are not able to reject the null hypothesis that the treatment, placebo training, and wait-list control groups differed. There were no significant differences between $\mathrm{ABM}$ training and placebo training or no training (waitlist) groups on pain symptoms. There were also no significant differences between ABM training, placebo training, or wait-list control groups on pain catastrophizing, generalized anxiety and depression symptoms, and physical functioning immediately after training or at 3-month follow-up. ABM training did not significantly impact attention bias or attention control in comparison to placebo training and no training; thus, the hypothesized training effect was not observed. Effect sizes for group comparisons between the ABM and placebo training condition from baseline to post-training were mostly small. There was a moderate effect size for the comparison of average pain level, however this (non-significant) effect was in the opposite direction than expected, with the ABM group actually reporting increased pain at post-training and the placebo group reporting reduced pain. Findings were similar across per-protocol and intention-to-treat analyses.

Our findings are somewhat inconsistent with previous findings demonstrating improved painrelated outcomes from ABM. Most relevant for comparison are two placebo-controlled studies that 
have investigated ABM in adults with chronic pain [9,53]. First, Carleton and colleagues [9] found that adult fibromyalgia patients reported reduced pain scores following ABM training. Second, Sharpe and colleagues [53] found that adult chronic pain patients reported improvements in functional disability following $\mathrm{ABM}$ training. In addition, both studies found improvements in a small number of pain-related cognitive-affective factors, including anxiety sensitivity $[9,53]$ and pain-related fear [9]. We did not replicate these findings with our sample of adolescents with chronic pain. A number of differences between the current and previous studies may explain inconsistencies. First, we did not measure anxiety sensitivity or pain-related fear; it may be that $\mathrm{ABM}$ influences only these specific constructs, or that training effects are stronger in subgroups of patients with high anxiety sensitivity or fear of pain. Thus these traits measured at baseline could moderate training effects. Second, we used a wider variety of training materials. Third, we administered fewer training trials per session, and fewer sessions overall than some prior adult ABM studies. ABM trials conducted in children and adolescents on mental health indices offer little consensus on the optimal number of sessions to enhance effectiveness [16]. Therefore, with the goal to maintain engagement among youth (and avoid boredom and fatigue), we decided in consultation with experienced clinicians to limit training to no more than 15 minutes, resulting in around 192 trials per session. Finally, participants in the current study completed all training sessions at home, whilst in previous studies training was completed partially or entirely in the clinic. It has been argued that at-home $\mathrm{ABM}$ training may be negatively influenced by the participant being in comfortable and familiar surroundings, whereas a level of mild anxiety, for example by visiting the clinic, is necessary for activating (and therefore changing) threat-related biases [8]. Completing the task at home may also have meant relatively less engagement with training by the participants due to the absence of an experimenter and/or the presence of uncontrolled distractions (although of note, we obtained high accuracy/fast response times). 
Some of our findings are consistent with other studies of ABM for chronic pain and psychopathology populations. First, there was no evidence of a pain-related attention bias at baseline in our sample, and no evidence that biases changed following training, thus replicating findings from Sharpe and colleagues [53]. This may explain our null findings. The absence of an attention bias at baseline could be due to a number of factors. A recent meta-analysis of attention biases in child and adolescent anxiety suggested that use of pictorial over linguistic stimuli and the presentation of 'threat' stimuli for longer durations (1000ms rather than 500ms) were variables that moderated the link between attention bias and anxiety [24]. Moreover, anxiety-associated attention biases were more likely to characterize older than younger youth, and other studies have also suggested complex interactions between attention control, attention bias and anxiety [21]. These methodological and sample considerations should be taken into account in future ABM trials to ensure a) the optimal assessment of biases, and b) that the sample selected manifest biases that could be shifted through training. Speaking to this, studies of ABM efficacy for anxiety and depression disorders indicate that effects on outcomes are most consistently found when biases exist at baseline [30] and when training successfully changes biases [13]. On the other hand, Sharpe and colleagues [53] demonstrated effects of ABM training on pain-related outcomes despite no change in attention bias. These discrepancies may be because of methodological issues, notably that the dot-probe task does not demonstrate good reliability as a measurement tool in pain populations [22], especially in children and adolescents where greater noise and variability may be present [20]. Changes in biases following ABM training, or a lack thereof, may therefore not be a reliable indicator of actual changes in attentional functioning. Our findings are also consistent with more recent $\mathrm{ABM}$ studies in anxiety and depression. Although meta-analyses of CBM in adults with anxiety have consistently found at least small but significant effects [17], the only childspecific meta-analysis has failed to find evidence of benefit in children and young people [16]. Where significant findings have been reported in individual trials, effect sizes are generally low, as 
demonstrated here as well. Given this, our trial is likely underpowered for the detection of these weak effects.

Nonetheless, our study design had significant advantages compared to previous studies on $\mathrm{ABM}$ in pain populations. First, word stimuli were idiosyncratically selected to match the quality of each participant's pain. Personal relevance of pain stimuli may be important for eliciting biases [21]. Therefore ABM training may be most efficacious when using such personalized stimuli. Second, we included two physical tasks (the sit-to-stand task and the cardio wall task) that provided objective measures of physical functioning. These tasks are frequently used in physiotherapy sessions and reflect measures that are used in clinical practice to make decisions about patients' treatment needs and progress. Third, given recent suggestion that $\mathrm{ABM}$ may impact attention control rather than valence-specific attentional selectivity [6], we considered the impact of ABM training on attention control, including both a self-report and an experimental measure. Although ABM training did not change attention control in our data,, future studies may consider including other measures of attention control, for example, the Attention Network Test (that is designed to test alerting, orienting, and executive control) [29], as well as antisaccade tasks (that are reliant on inhibitory eye-movement patterns) [36]. These other tasks could be designed to tap control of attention in pain-specific contexts rather than general trait abilities, as measured here, using the Attention Control Scale.

Findings presented here also add to our understanding of intervention delivery in pediatric chronic pain populations. First, recruitment of patients into the study was very successful: most patients approached by the team agreed to take part. Anecdotal evidence from the current study suggests that the computer-game interface was appealing to participants, suggesting that future interventions using a similar interface may achieve a similarly high recruitment success rate. Second, retention was high and while we collected data remotely, training indices revealed high levels of accuracy and fast response times. Subjective feedback also indicated moderate levels of 
task enjoyment and perceived usefulness of the training. This information is particularly important given current concerns over the face validity of computerized ABM training in adult samples [1]. Given the need for novel interventions that are both cost-effective and easy-to-access, we provide evidence that computerized training may be a promising platform for delivering interventions to youth with chronic pain. Of note, we also found improvements across all groups on a number of the task and questionnaire measures over time. These improvements could be explained in part by positive engagement with clinical services during assessments and by practice effects for the cognitive and physical tasks.

In conclusion, we report here the first randomized-controlled trial of $\mathrm{ABM}$ in a clinical sample of adolescents with chronic pain. We found no evidence that $\mathrm{ABM}$ affected pain symptoms compared with placebo training or wait-list control, or other pain-related outcomes. ABM also did not change attention bias or attention control in comparison with placebo training or no training, thus we did not find the predicted training effect. Calculated effect sizes on primary outcomes at post-intervention were also small. However, before we disregard ABM as a potential intervention, there are also a number of methodological reasons, and sample considerations that could have explained these null findings. Tailoring possible $\mathrm{ABM}$ to patients most likely to benefit from direct attentional re-direction (including recruitment of patients with more homogenous pain complaints) is a priority for future ABM trials. It may be that selecting those with extreme attentional biases, fear of pain, anxiety sensitivity and poor attention control would enable training effects to be observed more clearly. Other tools may also be needed to better shift attention biases. For example, given recent theory suggesting that attention biases are driven by biased interpretations of pain and pain-related information [19,58], and recent evidence that pain-related interpretation biases characterize adolescents with chronic pain [32], training tools that target interpretation biases (or other theoretical antecedents of attention bias) may also be useful for investigating the role of cognitive biases in pediatric chronic pain going forward [50]. 


\section{ACKNOWLEDGEMENTS}

The authors thank Tine Vervoort for providing the child face stimuli used in the attention bias measurement and training tasks, and Merel Koopmans for her assistance with study visits and data input. There are no conflicts of interest arising from this work.

\section{FIGURE LEGENDS}

Figure 1. CONSORT diagram illustrating the flow of participants through the study.

Figure 2. Example trial from the one of the ABM training tasks. The stimuli used during ABM were either faces (shown) or words. On each trial a fixation cross was presented, followed by two stimuli (pain/neutral or neutral/neutral), followed by a probe (p or q) to which the patients had to respond. During ABM (shown) the probe appeared behind the neutral stimulus; the placebo ABM condition was identical in every respect other than that the probe was equally likely to appear behind either the neutral or pain stimulus. Nb., stimuli are not presented to scale.

\section{REFERENCES}

[1] Beard C. Cognitive bias modification for anxiety: current evidence and future directions. Expert Rev. Neurother. 2011;11:299-311.

[2] Beck JE, Lipani T a., Baber KF, Dufton L, Garber J, Smith C a., Walker LS. Attentional bias to pain and social threat in pediatric patients with functional abdominal pain and pain-free youth before and after performance evaluation. Pain 2011;152:1061-1067. doi:10.1016/j.pain.2011.01.029.

[3] Bowler JO, Bartholomew KJ, Kellar I, Mackintosh B, Hoppitt L, Bayliss AP. Attentional bias modification for acute experimental pain: A randomized controlled trial of retraining 
early versus later attention on pain severity, threshold and tolerance. Eur. J. Pain 2016;21:113. doi:10.1002/ejp.908.

[4] Boyer MC, Compas BE, Stanger C, Colletti RB, Konik BS, Morrow SB, Thomsen AH. Attentional biases to pain and social threat in children with recurrent abdominal pain. J. Pediatr. Psychol. 2006;31:209-220.

[5] Browning M, Holmes EA, Murphy SE, Goodwin GM, Harmer CJ. Lateral Prefrontal Cortex Mediates the Cognitive Modification of Attentional Bias. Biol. Psychiatry 2010;67:919-925.

[6] Browning M, Holmes EA, Murphy SE, Goodwin GM, Harmer CJ. Lateral Prefrontal Cortex Mediates the Cognitive Modification of Attentional Bias. Biol Psychiatry 2010;67:919-925. doi:http://dx.doi.org/10.1016/j.biopsych.2009.10.031.

[7] Caes L, Vervoort T, Eccleston C, Vandenhende M, Goubert L. Parental catastrophizing about child's pain and its relationship with activity restriction: The mediating role of parental distress. Pain 2011;152:212-222. doi:10.1016/j.pain.2010.10.037.

[8] Carlbring P, Apelstrand M, Sehlin H, Amir N, Rousseau A, Hofmann SG, Andersson G. Internet-delivered attention bias modification training in individuals with social anxiety disorder - a double blind randomized controlled trial. BMC Psychiatry 2012;12:66. doi:10.1186/1471-244X-12-66.

[9] Carleton RN, Richter A a., Asmundson GJG. Attention Modification in Persons with Fibromyalgia: A Double Blind, Randomized Clinical Trial. Cogn. Behav. Ther. 2011;40:279-290.

[10] Chorpita BF, Moffitt CE, Gray J. Psychometric properties of the Revised Child Anxiety and Depression Scale in a clinical sample. Behav. Res. Ther. 2005;43:309-322.

[11] Chorpita BF, Yim L, Moffitt C, Umemoto L a., Francis SE. Assessment of symptoms of DSM-IV anxiety and depression in children: A revised child anxiety and depression scale. Behav. Res. Ther. 2000;38:835-855. 
[12] Christ SE, Kester LE, Bodner KE, Miles JH. Evidence for selective inhibitory impairment in individuals with autism spectrum disorder. Neuropsychology 2011;25:690-701.

[13] Clarke PJF, Notebaert L, MacLeod C. Absence of evidence or evidence of absence: reflecting on therapeutic implementations of attentional bias modification. BMC Psychiatry 2014;14:8. doi:10.1186/1471-244X-14-8.

[14] Cleeland CS, Ryan KM. Pain assessment: global use of the Brief Pain Inventory. Ann. Acad. Med. Singapore 1994;23:129-138.

[15] Cohen Kadosh K, Heathcote LC, Lau JYF. Age-related changes in attentional control across adolescence: how does this impact emotion regulation capacities? Front. Psychol. 2014;5:111. doi:10.3389/fpsyg.2014.00111.

[16] Cristea I a., Mogoașe C, David D, Cuijpers P. Practitioner Review: Cognitive bias modification for mental health problems in children and adolescents: a meta-analysis. J. Child Psychol. Psychiatry 2015:n/a-n/a. doi:10.1111/jcpp.12383.

[17] Cristea IA, Kok RN, Cuijpers P. Efficacy of cognitive bias modification interventions in anxiety and depression: meta-analysis. Br. J. Psychiatry 2015;206:7-16. doi:10.1192/bjp.bp.114.146761.

[18] Crombez G, Bijttebier P, Eccleston C, Mascagni T, Mertens G, Goubert L, Verstraeten K. The child version of the pain catastrophizing scale (PCS-C): A preliminary validation. Pain 2003;104:639-646.

[19] Crombez G, Heathcote LC, Fox E. The puzzle of attentional bias to pain : beyond attention. Pain 2015;156:1581-1582.

[20] Van Damme S, Crombez G. Measuring attentional bias to threat in children and adolescents: A matter of speed? J. Behav. Ther. Exp. Psychiatry 2009;40:344-351. doi:10.1016/j.jbtep.2009.01.004.

[21] Dear BF, Sharpe L, Nicholas MK, Refshauge K. Pain-related attentional biases: The 
importance of the personal relevance and ecological validity of stimuli. J. Pain 2011;12:625632. doi:10.1016/j.jpain.2010.11.010.

[22] Dear BF, Sharpe L, Nicholas MK, Refshauge K. The psychometric properties of the dotprobe paradigm when used in pain-related attentional bias research. J Pain 2011;12:12471254.

[23] Derryberry D, Reed MA. Anxiety-related attentional biases and their regulation by attentional control. J. Abnorm. Psychol. 2002;111:225-236.

[24] Dudeney J, Sharpe L, Hunt C. Attentional bias towards threatening stimuli in children with anxiety: A meta-analysis. Clin. Psychol. Rev. 2015;40:66-75.

[25] Eccleston C, Crombez G. Pain demands attention: a cognitive-affective model of the interruptive function of pain. Psychol. Bull. 1999;125:356-366.

[26] Eccleston C, Crombez G. Worry and chronic pain: A misdirected problem solving model. Pain 2007;132:233-236.

[27] Eldar S, Apter A, Lotan D, Edgar KP, Naim R, Fox NA, Pine DS, Bar-Haim Y. Attention bias modification treatment for pediatric anxiety disorders: a randomized controlled trial. Am. J. Psychiatry 2012;169:213-20.

[28] Esbjørn BH, Sømhovd MJ, Turnstedt C, Reinholdt-Dunne ML. Assessing the revised child anxiety and depression scale (RCADS) in a national sample of Danish youth aged 8-16 years. PLoS One 2012;7.

[29] Fan J, McCandliss BD, Sommer T, Raz A, Posner MI. Testing the efficiency and independence of attentional networks. J. Cogn. Neurosci. 2002;14:340-347.

[30] Fox E, Zougkou K, Ashwin C, Cahill S. Investigating the efficacy of attention bias modification in reducing high spider fear: The role of individual differences in initial bias. J. Behav. Ther. Exp. Psychiatry 2015;49:84-93.

[31] Gupta SK. Intention-to-treat concept: A review. Perspect. Clin. Res. 2011;2:109-112. 
doi:10.4103/2229-3485.83221.

[32] Heathcote LC, Jacobs K, Eccleston C, Fox E, Lau JYF. Biased interpretations of ambiguous bodily threat information in adolescents with chronic pain. Pain $2017 ; 158: 471-478$.

[33] Heathcote LC, Lau JYF, Mueller SC, Eccleston C, Fox E, Bosmans M, Vervoort T. Child attention to pain and pain tolerance are dependent upon anxiety and attention control: An eye-tracking study. Eur. J. Pain 2016;21:250-263.

[34] Heathcote LC, Vervoort T, Eccleston C, Fox E, Jacobs K, Van Ryckeghem DML, Lau JYF. The relationship between adolescents' pain catastrophizing and attention bias to pain faces is moderated by attention control. Pain 2015;156:1334-1341.

[35] Heeren A, De Raedt R, Koster EHW, Philippot P. The (neuro)cognitive mechanisms behind attention bias modification in anxiety: proposals based on theoretical accounts of attentional bias. Front. Hum. Neurosci. 2013;7:119. doi:10.3389/fnhum.2013.00119.

[36] Hutton SB, Ettinger U. The antisaccade task as a research tool in psychopathology: A critical review. Psychophysiology 2006;43:302-313.

[37] Kashikar-Zuck S, Flowers SR, Claar RL, Guite JW, Logan DE, Lynch-Jordan AM, Palermo TM, Wilson AC. Clinical utility and validity of the Functional Disability Inventory (FDI) among a multicenter sample of youth with chronic pain. Pain 2011;152:1600-1607. doi:10.1016/j.pain.2011.02.050.

[38] Koster EHW, Crombez G, Verschuere B, De Houwer J. Selective attention to threat in the dot probe paradigm: Differentiating vigilance and difficulty to disengage. Behav. Res. Ther. 2004;42:1183-1192.

[39] MacLeod C, Mathews A, Tata P. Attentional bias in emotional disorders. J. Abnorm. Psychol. 1986;95:15-20. doi:10.1037/0021-843X.95.1.15.

[40] MacLeod C, Soong LY, Rutherford EM, Campbell LW. Internet-delivered assessment and manipulation of anxiety-linked attentional bias: validation of a free-access attentional probe 
software package. Behav. Res. Methods 2007;39:533-538.

[41] Mathews A, Yiend J, Lawrence AD. Individual differences in the modulation of fear-related brain activation by attentional control. J. Cogn. Neurosci. 2004;16:1683-94. doi:10.1162/0898929042947810.

[42] Mathyssek CM, Olino TM, Hartman CA, Ormel J, Verhulst FC, Van Oort FVA. Does the Revised Child Anxiety and Depression Scale (RCADS) measure anxiety symptoms consistently across adolescence? The TRAILS study. Int. J. Methods Psychiatr. Res. 2013;22:27-35.

[43] McGowan N, Sharpe L, Refshauge K, Nicholas MK. The effect of attentional re-training and threat expectancy in response to acute pain. Pain 2009;142:101-107. doi:10.1016/j.pain.2008.12.009.

[44] Monk CS, McClure EB, Nelson EE, Zarahn E, Bilder RM, Leibenluft E, Charney DS, Ernst M, Pine DS. Adolescent immaturity in attention-related brain engagement to emotional facial expressions. Neuroimage 2003;20:420-428.

[45] Muris P, de Jong PJ, Engelen S. Relationships between neuroticism, attentional control, and anxiety disorders symptoms in non-clinical children. Pers. Individ. Dif. 2004;37:789-797.

[46] Nelson CA, Bloom FE, Cameron JL, Amaral D, Dahl RE, Pine D. An integrative, multidisciplinary approach to the study of brain-behavior relations in the context of typical and atypical development. Dev. Psychopathol. 2002;14:499-520.

[47] Perquin CW, Hazebroek-Kampschreur A a JM, Hunfeld J a M, Bohnen AM, Van SuijlekomSmit LW a, Passchier J, Van Der Wouden JC. Pain in children and adolescents: A common experience. Pain 2000;87:51-58.

[48] Pincus T, Morley S. Cognitive-processing bias in chronic pain: A review and integration. Psychol. Bull. 2001;127:599-617.

[49] Rueda MR, Fan J, McCandliss BD, Halparin JD, Gruber DB, Lercari LP, Posner MI. 
Development of attentional networks in childhood. Neuropsychologia 2004;42:1029-1040.

[50] Van Ryckeghem DML, Crombez G. Attentional bias and chronic pain: Where to go from here? Pain 2014;155:6-7. doi:10.1016/j.pain.2013.10.025.

[51] Van Ryckeghem DML, Crombez G, Van Hulle L, Van Damme S. Attentional bias towards pain-related information diminishes the efficacy of distraction. Pain 2012;153:2345-2351. doi:10.1016/j.pain.2012.07.032.

[52] Schoth DE, Georgallis T, Liossi C. Attentional bias modification in people with chronic pain: a proof of concept study. Cogn. Behav. Ther. 2013;42:233-43. doi:10.1080/16506073.2013.777105.

[53] Sharpe L, Ianiello M, Dear BF, Nicholson Perry K, Refshauge K, Nicholas MK. Is there a potential role for attention bias modification in pain patients? Results of 2 randomised, controlled trials. Pain 2012;153:722-731. doi:10.1016/j.pain.2011.12.014.

[54] Sharpe L, Johnson A, Dear BF. Attention bias modification and its impact on experimental pain outcomes: Comparison of training with words versus faces in pain. Eur. J. Pain 2015:n/a-n/a. doi:10.1002/ejp.648.

[55] Simmons JP, Nelson LD, Simonsohn U. False-Positive Psychology: Undisclosed Flexibility in Data Collection and Analysis Allows Presenting Anything as Significant. Psychol. Sci. 2011;22:1359-1366.

[56] Simon D, Craig KD, Gosselin F, Belin P, Rainville P. Recognition and discrimination of prototypical dynamic expressions of pain and emotions. Pain 2008;135:55-64.

[57] Spear LP. The adolescent brain and age-related behavioral manifestations. Neurosci. Biobehav. Rev. 2000;24:417-463.

[58] Todd J, Sharpe L, Johnson A, Nicholson K, Colagiuri B, Dear BF. Towards a new model of attentional biases in the development, maintenance, and management of pain. Pain 2015;156:1589-1600. 
[59] Todd J, Sharpe L, Johnson A, Nicholson Perry K, Colagiuri B, Dear BF. Towards a new model of attentional biases in the development, maintenance and management of pain. Pain 2015;156:1589-1600.

[60] Vervoort T, Caes L, Trost Z, Notebaert L, Goubert L. Parental attention to their child's pain is modulated by threat-value of pain. Heal. Psychol. 2012;31:623-631.

[61] Vervoort T, Trost Z, Van Ryckeghem DML. Children's selective attention to pain and avoidance behaviour: The role of child and parental catastrophizing about pain. Pain 2013;154:1979-1988. doi:10.1016/j.pain.2013.05.052.

[62] Vlaeyen JWS, Linton SJ. Fear-avoidance and its consequences in chronic musculoskeletal pain: A state of the art. Pain 2000;85:317-332.

[63] Walker LS, Greene JW. The functional disability inventory: measuring a neglected dimension of child health status. J. Pediatr. Psychol. 1991;16:39-58. 
Table 1. Baseline demographic and pain characteristics

\begin{tabular}{|c|c|c|c|c|}
\hline Measure & $\mathrm{ABM}(\mathrm{n}=23)$ & Placebo $(n=22)$ & Waitlist $(n=21)$ & Total $(n=66)$ \\
\hline \multicolumn{5}{|l|}{ Demographics } \\
\hline Sex [female] & $20(87.0 \%)$ & $17(77.3 \%)$ & $18(85.7 \%)$ & $55(83.3 \%)$ \\
\hline Age [years] & $13.48(2.15)$ & $13.95(2.04)$ & $14.52(2.18)$ & $13.97(2.13)$ \\
\hline \multicolumn{5}{|l|}{ Ethnicity } \\
\hline Caucasian & $21(91.3 \%)$ & $21(95.5 \%)$ & $19(90.5 \%)$ & $61(92.4 \%)$ \\
\hline Asian & $1(4.3 \%)$ & $1(4.5 \%)$ & $1(4.8 \%)$ & $3(4.5 \%)$ \\
\hline Other & $1(4.3 \%)$ & $0(0.0 \%)$ & $1(4.8 \%)$ & $2(3.0 \%)$ \\
\hline Has siblings & $21(91.3 \%)$ & $20(90.9 \%)$ & $18(85.7 \%)$ & $59(89.4 \%)$ \\
\hline \multicolumn{5}{|l|}{ Pain variables } \\
\hline Time since pain onset [months] & $44.70(34.58)$ & $39.18(23.66)$ & $53.48(45.25)$ & $45.65(35.35)$ \\
\hline Attends full time school & $19(82.6 \%)$ & $18(81.8 \%)$ & $14(66.7 \%)$ & $51(77.3 \%)$ \\
\hline Pain onset (gradual) & $13(56.5 \%)$ & $10(45.5 \%)$ & $16(76.2 \%)$ & $39(59.1 \%)$ \\
\hline Pain in more than one area & $21(91.3 \%)$ & $20(90.9 \%)$ & $17(81.0 \%)$ & $58(87.9)$ \\
\hline Pain at most problematic site [/10, last week] & $7.48(1.83)$ & $6.86(1.64)$ & $7.52(1.63)$ & $7.29(1.71)$ \\
\hline Average pain [/10, last week] & $6.09(1.86)$ & $6.18(1.89)$ & $6.33(2.06)$ & $6.20(1.91)$ \\
\hline Pain duration [/4, last week] & $3.17(0.94)$ & $3.39(0.87)$ & $3.67(0.66)$ & $3.39(0.87)$ \\
\hline
\end{tabular}

Categorical variables: $N(\%)$; Ordinal/continuous variables: Mean $(S D)$

$\mathrm{Nb}$. No significant differences between groups on any measures 
Table 2. Means and SDs of outcome and process measures for each group at each time point (ITT analyses)

\begin{tabular}{|c|c|c|c|c|c|c|c|c|c|}
\hline \multirow[t]{2}{*}{ Measure } & \multicolumn{3}{|l|}{ ABM } & \multicolumn{3}{|l|}{ Placebo } & \multicolumn{3}{|l|}{ Waitlist } \\
\hline & Baseline & Post-training & Follow-up & Baseline & Post-training & Follow-up & Baseline & Post-training & Follow-up \\
\hline \multicolumn{10}{|l|}{ Primary outcomes } \\
\hline $\begin{array}{l}\text { Pain at most problematic site } \\
\text { (last week; } 0-10 \text { ) }\end{array}$ & $7.48(1.83)$ & $7.17(1.85)$ & $7.00(2.11)$ & $6.86(1.64)$ & $6.95(1.59)$ & $6.23(1.95)$ & $7.52(1.63)$ & $7.05(2.01)$ & $7.00(2.14)$ \\
\hline Average pain (last week; 0-10) & $6.09(1.86)$ & $6.57(1.67)$ & $5.39(2.33)$ & $6.18(1.89)$ & $5.91(0.43)$ & $5.50(1.92)$ & $6.33(2.06)$ & $6.24(2.00)$ & $6.48(2.46)$ \\
\hline Pain duration (last week; 1-4) & $3.17(0.94)$ & $2.91(1.04)$ & $2.61(1.20)$ & $3.39(0.87)$ & $3.14(1.04)$ & $3.18(1.10)$ & $3.67(0.66)$ & $3.48(0.19)$ & $3.38(1.02)$ \\
\hline \multicolumn{10}{|l|}{ Secondary outcomes } \\
\hline Disability (FDI; 0-60) & $22.14(10.70)$ & $19.35(10.01)$ & $15.65(10.28)$ & $23.90(12.29)$ & $19.73(13.31)$ & $19.18(12.08)$ & $22.69(9.64)$ & $19.64(9.89)$ & $17.43(9.91)$ \\
\hline $\begin{array}{l}\text { Pain catastrophizing (PCSC; } 0- \\
\text { 52) }\end{array}$ & $28.87(10.96)$ & $26.52(10.05)$ & $21.43(11.91)$ & $30.41(10.30)$ & $24.91(11.77)$ & $24.27(10.78)$ & $24.95(8.44)$ & $20.76(10.63)$ & $19.86(13.44)$ \\
\hline $\begin{array}{l}\text { Depression (RCADS- } \\
\text { Depression; 0-40) }\end{array}$ & $12.17(6.40)$ & $11.52(6.49)$ & $9.43(5.70)$ & $12.73(6.28)$ & $12.09(7.07)$ & $11.41(5.94)$ & $10.24(5.12)$ & $9.81(5.91)$ & $10.24(6.88)$ \\
\hline $\begin{array}{l}\text { Generalised anxiety (RCADS- } \\
\text { GAD; } 0-24 \text { ) }\end{array}$ & $7.97(4.34)$ & $6.65(3.96)$ & $6.04(4.54)$ & $7.36(4.14)$ & $6.32(3.88)$ & $6.59(3.51)$ & $5.38(4.01)$ & $5.86(3.82)$ & $4.71(4.10)$ \\
\hline Attention control (ACS; 20-80) & $47.13(8.22)$ & $50.26(8.93)$ & $51.78(10.49)$ & $47.82(9.14)$ & $48.14(9.50)$ & $48.56(10.85)$ & $49.55(8.42)$ & $50.57(10.93)$ & $51.43(11.18)$ \\
\hline Sit-to-stand task & $12.57(3.73)$ & $13.13(3.92)$ & $14.17(4.03)$ & $14.77(4.56)$ & $14.77(5.01)$ & $16.23(5.94)$ & $13.90(4.10)$ & $15.48(3.74)$ & $15.21(3.71)$ \\
\hline Cardio wall task & $45.26(11.60)$ & $49.70(13.79)$ & $52.48(12.72)$ & $48.36(14.08)$ & $52.91(16.24)$ & $53.18(14.64)$ & $49.43(16.11)$ & $56.10(13.41)$ & $55.14(14.25)$ \\
\hline \multicolumn{10}{|l|}{ Dot-probe task } \\
\hline Congruent trials (RT) & $651.54(125.25)$ & $564.01(141.62)$ & $539.31(89.38)$ & $630.89(124.35)$ & $537.65(92.77)$ & $534.51(91.19)$ & $659.62(112.32)$ & $625.28(125.14)$ & $589.48(113.93)$ \\
\hline Incongruent trials $(\mathrm{RT})$ & $651.19(129.62)$ & $557.37(128.63)$ & $540.88(91.39)$ & $627.00(127.31)$ & $534.35(97.37)$ & $531.65(88.64)$ & $661.38(114.08)$ & $617.17(120.39)$ & $587.90(112.54)$ \\
\hline \multicolumn{10}{|l|}{ Fish Flanker task } \\
\hline Congruent trials (RT) & $544.67(81.64)$ & $571.66(139.08)$ & $515.63(92.92)$ & $531.13(131.74)$ & $501.69(129.29)$ & $495.72(89.07)$ & $553.47(123.27)$ & $552.06(107.12)$ & $525.49(94.38)$ \\
\hline Incongruent trials (RT) & $588.61(90.93)$ & $612.65(147.01)$ & $564.60(99.51)$ & $551.77(122.45)$ & $545.66(140.33)$ & $530.67(104.95)$ & $602.05(161.94)$ & $601.40(119.24)$ & $562.91(109.22)$ \\
\hline
\end{tabular}

FDI = Functional Disability Index; PCS-C = Pain Catastrophizing Scale - Child; ACS = Attention Control Scale; RT = Response Time

$\mathrm{Nb}$. Measure ranges noted in brackets next to each measure name. 
Table 3. ANOVA results for all outcome measures (ITT analysis).

\begin{tabular}{|c|c|c|c|c|c|}
\hline Measure & Effect & $d f$ & $F$ & $p$ & 2 \\
\hline \multicolumn{6}{|l|}{ Primary outcomes } \\
\hline \multirow[t]{3}{*}{ Pain at most problematic site } & Time & $1.93,121.73$ & 2.66 & .08 & .04 \\
\hline & Group & 2,63 & 0.89 & .42 & .03 \\
\hline & Time x Group & $3.86,121.73$ & 0.43 & .78 & .01 \\
\hline \multirow[t]{3}{*}{ Average pain (last week) } & Time & $1.70,106.78$ & 2.30 & .11 & .04 \\
\hline & Group & 2,63 & 0.45 & .64 & .01 \\
\hline & Time x Group & $3.39,106.78$ & 1.82 & .14 & .06 \\
\hline \multirow[t]{3}{*}{ Pain duration (last week) } & Time & $1.88,118.54$ & 4.58 & .01 & .07 \\
\hline & Group & 2,63 & 2.96 & .06 & .09 \\
\hline & Time x Group & $3.76,118.54$ & 0.61 & .65 & .02 \\
\hline \multicolumn{6}{|l|}{ Secondary outcomes } \\
\hline \multirow[t]{3}{*}{ FDI } & Time & $1.83,115.20$ & 19.79 & .00 & .24 \\
\hline & Group & 2,63 & 0.20 & .82 & .01 \\
\hline & Time x Group & $3.66,115.20$ & 0.56 & .67 & .02 \\
\hline \multirow[t]{3}{*}{ PCS-C } & Time & $1.70,107.13$ & 16.85 & .00 & .21 \\
\hline & Group & 2,63 & 1.38 & .26 & .04 \\
\hline & Time x Group & $3.40,107.13$ & 0.97 & .42 & .03 \\
\hline \multirow[t]{3}{*}{ RCADS-Depression } & Time & $1.71,107.58$ & 4.74 & .02 & .07 \\
\hline & Group & 2,63 & 0.61 & .55 & .02 \\
\hline & Time x Group & $3.42,107.58$ & 1.99 & .11 & .06 \\
\hline \multirow[t]{3}{*}{ RCADS-Generalised anxiety } & Time & $1.64,103.13$ & 6.20 & .01 & .09 \\
\hline & Group & 2,63 & 1.15 & .32 & .04 \\
\hline & Time x Group & $3.27,103.13$ & 2.09 & .10 & .06 \\
\hline \multirow[t]{3}{*}{ Sit-to-stand task } & Time & $1.69,106.36$ & 8.75 & .00 & .12 \\
\hline & Group & 2,63 & 1.50 & .23 & .05 \\
\hline & Time x Group & $3.38,106.36$ & 1.30 & .28 & .04 \\
\hline \multirow[t]{2}{*}{ Cardio wall task } & Time & $1.90,119.45$ & 15.08 & .00 & .19 \\
\hline & Group & 2,63 & 0.63 & .53 & .02 \\
\hline
\end{tabular}




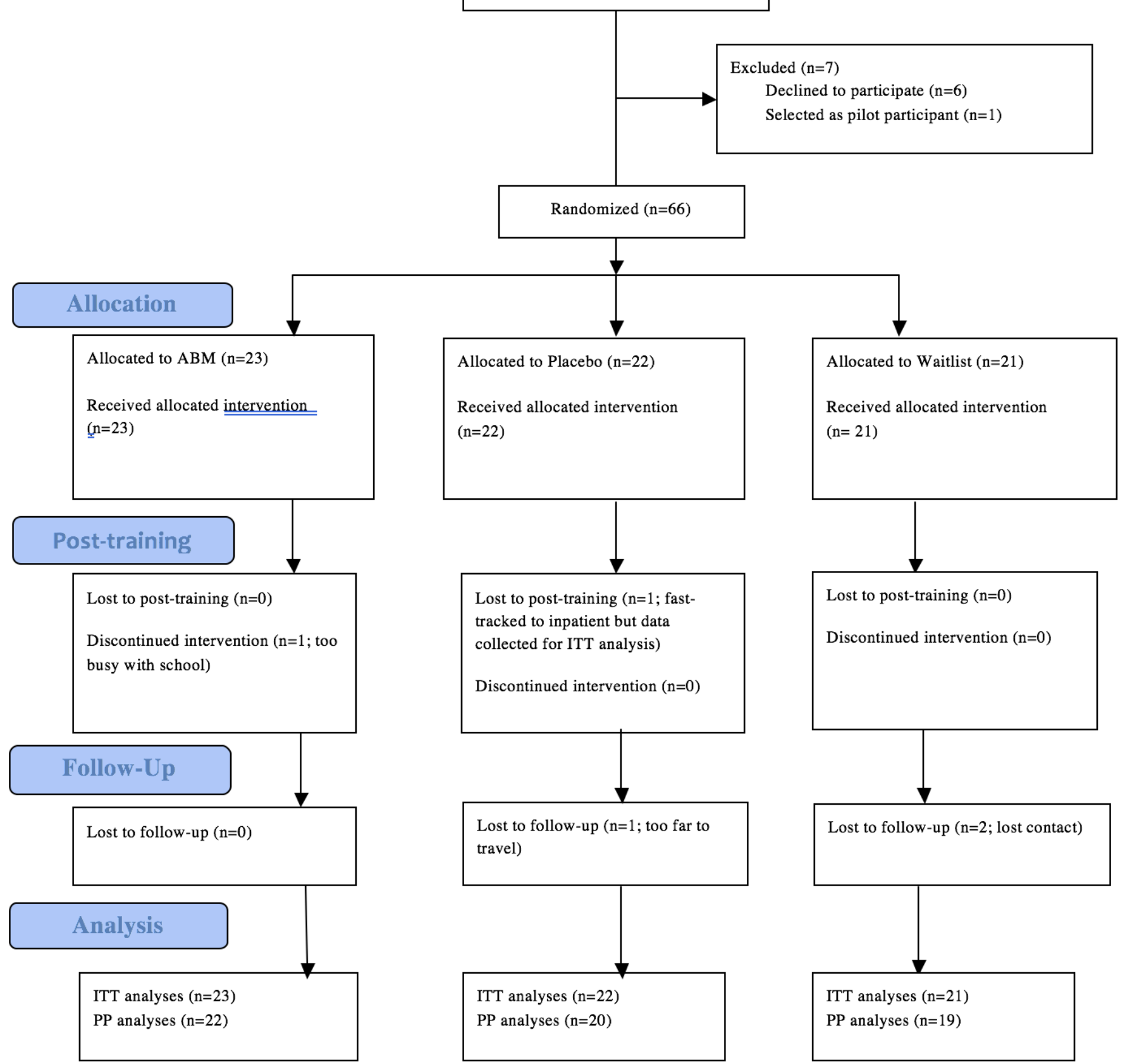

Figure 1 

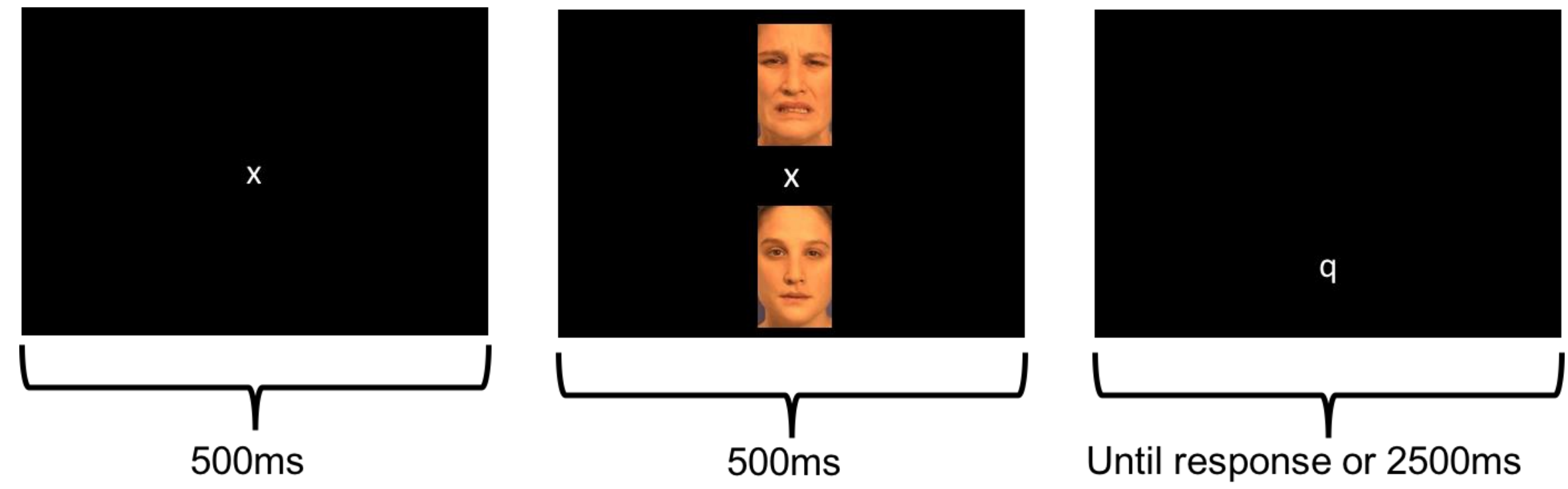

Until response or $2500 \mathrm{~ms}$

Time

Figure 2 